\title{
Lo strappo nella coscienza del lettore (spettatore) in Pirandello (e Pasolini)
}

\author{
Fulvio Orsitto \\ California State University \\ orsitto@gmail.com \\ https://dx.doi.org/10.12795/futhark.2013.i08.12
}

\begin{abstract}
This study has a twofold purpose: on one hand, it aims at confirming the importance of Pier Paolo Pasolini as one of the most relevant representatives of the postmodern trend in Italy and, on the other, it aims at liberating Luigi Pirandello's figure from the accusation of being an innovator only for the content of his works, but not for the language he chose to write them. While analyzing Pirandello's influence over Pasolini's oeuvre, this essay intends to demonstrate how the Sicilian author was certainly a modernist, but not an 'authoritarian modernist'. Indeed, his characters will never cross the threshold his works opened. Nonetheless, they will never return to the established order of things they have irrevocably altered. What is left of their existence will take place in an elsewhere, in a liminal zone near those horizons they helped opening; horizons later to be explored by postmodern narratives. This analysis is mainly based on Pirandello's The Late Mattia Pascal and Pasolini's What are the Clouds?, a short included in the film Caprice Italian Style [1968].
\end{abstract}

Keywords: Pirandello - The Late Mattia Pascal - One, No One And One Hundred Thousand - Modernism - Novel Of The 1900 - Pasolini - Postmodernism - Cinema - Film Studies - Short Film - Cinema And Literature Narrative Models - Narratology

Il presente saggio intende, da un lato, confermare l'importanza critica della figura di Pier Paolo Pasolini come uno dei principali rappresentanti di un 
certo tipo di sensibilità postmoderna in ambito italiano e, dall'altro, liberare in qualche modo la figura di Luigi Pirandello dall'etichetta di innovatore nel contenuto, ma non nella forma. Nel corso dello spazio dedicato dal presente studio all'influenza di quest'ultimo sull'opera pasoliniana si cercherà pertanto di dimostrare come quest'autore - pur appartenendo senza dubbio all'orizzonte modernista - non sia un 'modernista autoritario'. Se è pur vero, infatti, che i suoi personaggi non attraverseranno mai la soglia che le sue opere hanno contribuito ad aprire, è altrettanto vero che da parte loro non vi sarà neppure un ritorno all'ordine costituito. La parte rimanente della loro esistenza avrà luogo in una sorta di altrove, in una zona liminale comunque vicina a quegli orizzonti che hanno contribuito a dischiudere, e che spetterà poi ai personaggi figli della narrativa postmoderna esplorare. La presente analisi si basa soprattutto su II fu Mattia Pascal, per quanto concerne Pirandello, e su Che cosa sono le nuvole, cortometraggio contenuto nel film a episodi Capriccio all'italiana [1968], per quanto riguarda Pasolini.

Keywords: Pirandello - II Fu Mattia Pascal - Uno, Nessuno E Centomila Modernismo - Romanzo Del 900 - Pasolini - Postmodernismo - Cinema Cortometraggio - Rapporti Tra Cinema E Letteratura - Modelli Narrativi

\begin{abstract}
"As many postmodern theorist have tried to point out, the contemporary era is an era in which we must consistently confess our feelings: we answer magazine questionnaires about what we want, surveys about which politicians we like, focus groups about how we react to advertising campaigns; televised sport, war, accident and crime are all designed to trigger emotion. [...] things and events are now understood on the level of the pulsing, breathing, feeling individual self. Yet at the same time, this self is reported to feel les confident, more isolated, fragile and vulnerable than ever. [...] It is this ambivalence and ambiguity - the intensification of the self as the key site of human experience and its increasing sense of internal fragmentation and chaos - that the twentieth century's theorists of subjectivity have tried to deal with" (Mansfield 1-2).
\end{abstract}

Come ribadito da questa citazione di Nick Mansfield, e come affermato in precedenza negli scritti di Lyotard e Jameson, l'idea della soggettività che si è andata sviluppando in ambito postmoderno non si avvale più delle cosiddette 'cognitive maps' (Jameson) o delle 'grand narratives' (Lyotard) che miravano a rassicurare il soggetto, aiutandolo a definire se stesso. Per contro, dato che "we now live in a world where the only values present have been discredited, without having been replaced by anything else" (Mansfield 168), compito dell'autore postmoderno è proprio quello di superare il tentativo, comunque autoritario, del modernismo "to reinvent new and absolute principles by which we could live" (Mansfield 163). In un'ottica postmoderna, infatti, anche questo progetto utopico è andato in frantumi, ed ormai non resta che prendere atto - in chiave ottimistica - del fatto che "postmodernism frees up the infinite field of differences within humanity" oppure - secondo una lettura più pessimista 
- riconoscere che siamo stati abbandonati "in a junk-yard of values" (Mansfield 168).

Alla luce di queste considerazioni il seguente saggio intende quindi, da un lato, confermare l'importanza critica della figura di Pier Paolo Pasolini come uno dei principali rappresentanti di questo tipo di sensibilità postmoderna ${ }^{1}$ in ambito italiano e, dall'altro, liberare in qualche modo la figura di Luigi Pirandello dall'etichetta di innovatore nel contenuto ma non nella forma. Nel corso dello spazio dedicato dal presente studio all'influenza di quest'ultimo sull'opera pasoliniana si cercherà pertanto di dimostrare come quest'autore - pur appartenendo senza dubbio all'orizzonte modernista - non sia un 'modernista autoritario'. Se è pur vero, infatti, che i suoi personaggi non attraverseranno mai la soglia che le sue opere hanno contribuito ad aprire, è altrettanto vero che da parte loro non vi sarà neppure un ritorno all'ordine costituito. La parte rimanente della loro esistenza avrà luogo in una sorta di altrove, in una zona liminale comunque vicina a quegli orizzonti che hanno contribuito a dischiudere, e che spetterà poi ai personaggi figli della narrativa postmoderna esplorare. Per ragioni di brevitas la presente analisi verterà soprattutto su II fu Mattia Pascal, per quanto concerne Pirandello, e su Che cosa sono le nuvole, cortometraggio contenuto nel film a episodi Capriccio all'italiana [1968], per quanto riguarda Pasolini.

Durante la lettura del romanzo pirandelliano è come se, in qualche modo, assistessimo ad una lunga seduta di analisi alla ricerca dell'lo, dove il protagonista cerca continuamente di liberarsi dai fastidiosi vincoli sociali e di sottrarsi all'opprimente dominio dei luoghi comuni, rendendosi però conto alla fine che né Mattia né Adriano possono riuscire a spezzare quella concatenazione di legami e di oppressioni. A compiere questa serie di disperati tentativi è un soggetto che non trova di meglio che definirsi con la formula pseudo-giuridica di fu-Mattia-Pascal, rivelando la sua essenza di lo diviso, ma anche di lo multiplo, di lo in cerca di se stesso, o forse - ante litteram - in cerca del suo autore.

Come ricorda Dombroski, "Pirandello ci presenta la storia di Mattia che, a sua volta, ci racconta la storia di se stesso, o meglio, di Mattia Pascal, Adriano Meis, e del fu Mattia Pascal" (Dombroski 38). Tra i per-

Infatti, esaminando le varie tappe della carriera letteraria e cinematografica di quest'autore, è davvero possibile rendersi conto di come sia passato dal primo tipo di approccio ottimistico (si pensi al tentativo di riscoperta del passato attuato ne II vangelo secondo Matteo [1964], in Edipo re [1967], Medea [1969] e nella trilogia della vita (II Decameron [1971], I racconti di Canterbury [1972], II fiore delle mille e una notte [1974]) ed alla multiculuralità in embrione degli Appunti per un'orestiade africana [1969-1973] a quello più amaro e pessimista che culminerà nel primo capitolo della mai terminata trilogia della morte (ossia Salò o le 120 giornate di Sodoma [1975]). 
sonaggi incontrati dal narratore-protagonista spicca la figura di Anselmo Paleari, una sorta di filosofo teosofo - o filosofo affittuario, come suggerisce ironicamente Dombroski - che gli propone delle alternative che si rivelano ancora più tragicamente e comicamente inadatte ad una lettura della realtà. Né teosofia, né lanterninosofia, né spiritismo consentono a Mattia-Adriano di de-codificare il proprio lo. Paleari però all'inizio del XII capitolo, in uno dei ripetuti tentativi di aprire una conversazione, solleva un interrogativo che ripropone ed amplifica uno dei temi chiave della poetica pirandelliana e dell'intero modernismo, ossia l'instabilità dell'identità umana, e lo fa domandandosi:

"Se, nel momento culminante, proprio quando la marionetta che rappresenta Oreste è per vendicare la morte del padre sopra Egisto e la madre, si facesse uno strappo nel cielo di carta del teatrino, che avverrebbe?" (Pirandello 2003a, 100).

Renato Barilli, a commento delle meditazioni palearesi, afferma che "lo strappo o lo sfondamento significano l'avvenimento principe dell' universo pirandelliano, il passaggio all' «altra» dimensione, diversa da quella di superficie del buon senso" (Barilli 196). Tesi, questa, supportata e perfezionata anche da Robert Dombroski, il quale non manca di aggiungere che "il passaggio all' «altra dimensione» non è solo «l'avvenimento principe» in senso contenutistico o vagamente tematico (polemica contro il buon senso e l'appropriatezza borghese), ma è anche, e soprattutto, il principio che regola la composizione del romanzo" (Dombroski 45). É innegabile, del resto, che il movimento della narrazione proceda proprio 'a strappi', ossia attraverso una concatenazione di eventi esteriori procurati dal caso (che spesso hanno attirato sul romanzo critiche motivate da una presunta inverosimiglianza) come i due suicidi o la vincita a Montecarlo.

Lo strappo nel cielo di carta del teatrino quindi, in senso traslato, non significa "nient'altro che interrompere il filo della causalità logica, mettere in risalto l'imprevedibile come costante prevedibile dell'esistenza umana, cioè infrangere la dipendenza" (Dombroski 45$){ }^{2}$ Se però da un lato l'itinerario del protagonista è caratterizzato dalla liberazione graduale dalla schiavitù delle cose, dall'altro quest'ultimo finisce con il cadere vittima di un altro tipo di schiavitù quasi ontologica, e forse ancora più bieca, che lo costringe a rinunciare all'amore o anche solo all'affetto di un cane. In altre parole, Oreste non riuscirà mai a trasformarsi in Amleto, e quello strappo nel cielo di carta del teatrino di marionette non consenti-

2 Dombroski, peraltro, prosegue nel suo ragionamento affermando che "gli effetti funzionali di questa tecnica di scomposizione (umoristica) sono omologhi a quelli tipici dell'espressione del motto di spirito: scarico della tensione accumulata, eccitazione, e svalutazione della struttura sociale, effetti questi tutti presenti significativamente nella struttura dell'intreccio" (Dombroski 45). 
rà che una accettazione limitata di sè al fu-Mattia-Pascal, il quale potrà solo tornare a 'scrivere' la sua vita.

Le figure della prima parte della vita del narratore-protagonista alla fine non potranno che ritornare, secondo un ordine gerarchicamente diverso, metabolizzate attraverso le vite di Adriano e Mattia a ricordargli che, nella sua solitudine, può solo affermare l'esistenza di un fu-MattiaPascal. Secondo Pirandello quindi, non è possibile ribellarsi al destino. Se ne può sentire la necessità, ma sapendo quanto sia problematico prendere le distanze dai condizionamenti che soffocano l'individuo e lo fanno schiavo delle convenzioni, al soggetto non rimane che dichiararsi consapevole testimone (oltre che protagonista) della tragicomica limitatezza dell'uomo nell'universo.

Pirandello quindi si configura come artista e "uomo del passaggio" secondo la definizione di Anna Ossani - ma anche "raffinato e sadico 'logoteta', tragico burattinaio, la cui koinè greco-sicula e insieme tedesca, lo spinge verso l'amara e lucida scoperta 'dello strappo nel cielo di carta' delle proprie pagine, ma là dove la stanza della tortura è più orrenda, la segreta più buia" (Ossani 14-15). Questo romanzo finisce quindi anche con il rivelare la nuova funzione che l'arte avrà per quest'autore: "fuggire dalla contingenza storica mediante la riflessione umoristica sull'Essere" (Dombroski 50).

Tra le critiche mosse a II fu Mattia Pascal, si distingue quella di Giacomo Debenedetti, il quale anzichè attaccare i vari processi di astrazione e simbolizzazione (la paratassi rifiutata da Lukàcs), si interroga sul desiderio del protagonista "di ritrovarsi come persona fuori del contesto dei rapporti umani, mentre allo stesso tempo egli ritorna a quella identica struttura sociale da cui aveva tentato di esiliarsi" (Debenedetti 341). Ebbene, occorre innanzitutto sottolineare che le riserve avanzate da Debenedetti non tengono conto del fatto che è lo stesso narratoreprotagonista ad affermare "non sono affatto rientrato nè nella legge, nè nelle mie particolarità. Mia moglie è moglie di Pomino, ed io non saprei proprio dire ch'io mi sia" (Pirandello 2003a, 172). Quest'ultimo poi corregge ancora il tiro, e ribadisce la sua esistenza in una liminalità proprio nelle ultime righe, in cui oltre a riferirci "socchiudo gli occhi", conclude con la nota definizione ossimorica "io sono il fu Mattia Pascal" (Pirandello 2003a, 172). Più appropriate paiono invece le osservazioni di Roberto Alonge, il quale sottolinea "l'incapacità di andare oltre, di rompere definitivamente con le proprie paure, le proprie angosce" (Alonge 154), concludendo che II fu Mattia Pascal è "il romanzo dell'impossibilità del piccolo borghese di uscire dai limiti del proprio ceto medio, di diventare altro, non piccolo borghese" (Alonge 161).

Verso un uomo senza maschera, un soggetto puro, tende utopicamente anche un altro romanzo pirandelliano, quell'Uno nessuno e centomila in cui il protagonista, Vitangelo Moscarda, finisce con il rifiutare "la 
sua "maschera», tutto ciò che è personale, con un'azione opposta a quella da cui la sua storia aveva avuto inizio" (Biasin 62). In questo caso però l'io moscardiano finisce con l'inglobare tutto: "sono quest'albero. Albero, nuvola; domani libro o vento: il libro che leggo, il vento che bevo. Tutto fuori, vagabondo" e poi ancora "muoio ogni attimo, io, e rinasco nuovo e senza ricordi: vivo e intero, non più in me, ma in ogni cosa fuori" (Pirandello 2003b, 772). Tutto, quindi, pare esistere al di fuori del tempo e dello spazio. Per vivere pare necessario annullarsi. Privato della sua identità specifica ed autonoma con l'immersione nel paesaggio, Moscarda perde quello che era il suo lo, ma così facendo non fa che iniziare una nuova meccanismo di fictio, in cui a dominare sarà il non-Essere anzichè l'Essere. In altre parole, 'l'esperienza di Moscarda è basata sulla convinzione che vivere significa, necessariamente, recitare, giocare una parte, fingersi" (Dombroski 134) ed in questo modo non fa che inserirsi nella vasta schiera di personaggi pirandelliani che raggiungono la lucida consapevolezza di stare recitando una parte. Conditio sine qua non di questa 'meta-recita' è, secondo Dombroski, il "convincersi della realtà di un fatto o di una cosa," perchè "il loro modo di vincere nel conflitto coll'altro è di tentare sempre un'ultima finzione, di creare ancora l'ultima verità che riguarda se stessi, al fine di eludere la realizzazione semplice e diretta della loro realtà" (Dombroski 134).

Strettamente connesso a questa nuova visione della realtà troviamo sicuramente l'atteggiamento umoristico, ${ }^{3}$ ma anche quella apparentemente arbitraria digressione su Copernico che fa da premessa filosofica a Il fu Mattia Pascal. Se, infatti, togliere all'uomo la sua posizione di predominanza (di centro dell'universo) implica la distruzione di ogni residua illusione di supremazia (denunciando la sua incapacità di cogliere verità assolute), per contro va altresì sottolineato come questa situazione contribuisca a ridefinirlo come artefice di una nuova entità relazionale. ${ }^{4}$

L'uomo antico, insomma, ben saldo nelle sue finte certezze, agiva senza dubbi o ripensamenti, proprio perchè il suo orizzonte era chiuso

3 A questo proposito Auréliu Weiss afferma che "dans ce théâtre qui remet en discussion toutes nos certitudes, la consistance et l'unité de notre caractère, la possibilité de communiquer aux autres le sens exact de nos pensèes et de nos sentiments, la valeur des vérités humaines, l'image que nous nous formons de la personnalité des autres conditionnant pratiquement leur vie....ien ne reste debout : toutes paraissent chanceler, prêtes à s'effondrer. La communion apparente des hommes se change en mésentente, l'évidence en doute, la vérité en erreur, la connaissance en néant. L'humorisme n'est qu'un instrument de plus dans ce jeu qui tend à renverser nos certitudes" (Weiss 10).

4 Per una più approfondita trattazione del rapporto tra "Mattia Pascal's «reading eye», juxtaposed with his "wandering eye»" e per altre acute riflessioni "on the implications of a monocular point of view" si veda Donatella Stocchi Perucchio, Pirandello and the Vagaries of Knowledge. A Reading of II fu Mattia Pascal. Saratoga: ANMA Libri and Dept. Of French and Italian Stanford University, 1991. 
dal cielo di carta. L'uomo moderno, invece, distratto e sconcertato da quello 'strappo nel cielo di carta', ossia da una vita in cui è finalmente in grado di distinguere i condizionamenti che lo soffocano e lo rendono schiavo delle convenzioni, è dibattuto tra mille dubbi ed incertezze, combattuto (come Amleto) tra l'Essere ed il Non-Essere. A differenza delle marionette - che possono recitare tranquillamente la loro parte nella 'commedia della vita', protette da quel 'cielo di carta' che si conserva senza strappi - il narratore-protagonista, dopo un periodo di libertà quanto mai illusoria, viene ripreso dai dubbi e dalle paure, da tutte quelle angosciose perplessità, a cui invece nella loro incoscienza esse sono immuni.

Mattia-Adriano, pertanto, è il simbolo della "differenza fra la tragedia antica e moderna" (Pirandello 2003a, 100), nonchè la vittima principale di uno 'strappo' che lo fa soffrire proprio in quanto lo rende partecipe della sua triste condizione esistenziale e, ancor di più, della sostanziale impossibilità di porvi rimedio. Dopo essere morto due volte, e due volte ritornato in vita, alla fine è più solo di prima, senza una identità precisa, anzi, costretto a misurarsi con l'impossibilità di stabilire una soggettività che non sia transitoria o liminale, senza poter neppure dire: io sono Mattia Pascal.

$\dot{E}$ proprio in questi aspetti che va quindi ricercata la modernità, o 'postmodernità' - intesa come anticipazione del postmoderno - di Pirandello. ${ }^{5}$ L'enfasi pirandelliana sulle vane apparenze che circondano l'uomo non fa quindi che confermarci quanto, secondo Auréliu Weiss, "la vie lui apparaissait comme une scène immense sur laquelle des personnages affublés de masques grotesques jouaient des rôles pour lesquels ils n'étaient pas faits; drame silencieux qui échappait à la conscience des acteurs, jusqu'à ce qu'une douleur poignante, débordant les convenances routinières, vienne arracher violemment les masques et mettre à nu les visages" (Weiss 13). Che cosa è infatti questa voglia di liberarsi della maschera se non l'equivalente di uno stare a contemplare lo 'strappo nel cielo di carta'?

È vero che nè il narratore-protagonista de /l fu Mattia Pascal nè il Vitangelo Moscarda di Uno, nessuno e centomila attraverseranno mai questa soglia, ma è altrettanto vero che sono stati loro - in qualche modo - ad aprirla, e che ormai per loro è impossibile un ritorno all'iniziale stato delle cose. La liminalità nella quale decidono di condurre il resto della loro esistenza non può che segnare la sconfitta di una epistemologia

A questo proposito, ed a conferma dell'intuizione pirandelliana, è ancora Mansfield a ricordarci che se esiste una cosa su cui tutte le "theories of subjectivity that have dominated the last thirty years of literary and cultural studies" concordano è proprio il fatto che "they reject the idea of the subject as a completely self-contained" (Mansfield 13). 
positivista a vantaggio di un significativo prodromo dello scetticismo postmoderno. Infatti, la formula paradossale con cui si conclude il romanzo sancisce, secondo Donatella Stocchi Perucchio, "the disillusion about the possibility of rebirth, and also the failure to retrieve, under the mask of Adriano Meis, the authentic Mattia Pascal. [...] the exordium about naming, «mi chiamavo Mattia Pascal» is mirrored here by a conclusion about being, «io sono il fu Mattia Pascal». The essence of being is reduced to the name, which is a remnant, as the temporal paradox indicates, of a radical loss of foundation" (Stocchi Perucchio 41).

Alla conclusione dei romanzi, ma anche del teatro di Pirandello, abbiamo quindi la mancanza di un'unica fictio universalmente valida. Quello che abbiamo, per contro, è frammentazione, è una moltiplicazione plurima dei processi e dei costrutti fictionali in quanto, come sostiene Manuela Gieri, "the new Subject can no longer express himself in traditional linguistic and social ways. The new Subject is anarchic in his very spirit, and hence restless towards any given form" (Gieri 155).

Secondo Roland Barthes, in un'età come la nostra "the death of the Father would deprive literature of many of its pleasures. If there is no longer a Father, why tell stories? Doesn't any narrative lead back to Oedipus? Isn't storytelling always a way of searching for one's origin, speaking one's conflict with the Law, entering into the dialectic form of tenderness and hatred? Today, we dismiss Oedipus and narrative at one and the same time: we no longer love, we no longer fear, we no longer narrate" (Barthes 47). Di conseguenza, la ricetta pirandelliana per recuperare 'the pleasure principle' pare risolversi - ad una prima lettura - in una specie di ritorno a quella che Lacan, in The Mirror Stage, definisce "a jubilant assumption of his specular image" (Lacan 2), ossia un reditus al momento in cui il soggetto non era ancora conscio dello 'strappo' ed esposto ad una conseguente alienazione. Ad un'analisi più attenta, però, non può sfuggire l'enfasi dell'autore sulla "reaction against false concepts of «normality» both in art and life, to which it opposes first «abnormality», and then «transgression», beyond the given laws and norms" (Gieri 156), evidenziando quindi soprattutto quell'aspetto di moltiplicazione della fictio che costituisce uno degli aspetti più innovativi dell'opera pirandelliana.

"Il vero obiettivo che il cineasta dovrebbe proporsi nella sua scelta di filmare un testo letterario, non è un film paragonabile al romanzo, o degno di lui, ma un nuovo oggetto estetico che è qualcosa come il romanzo moltiplicato per il cinema" (Andrè Bazin, in Manzoli 93).

Tra i vari 'strappi' vissuti dall'opera pirandelliana e, in questo caso, dalla letteratura in generale, ve ne è uno i cui effetti sono stati, e sono tuttora, di portata enorme. Si tratta dello 'strappo' che ha messo in contatto due mondi, due strutture fictionali come il cinema ed, appunto, la letteratura. 
Anche questa volta - come sottolineato dalla precedente citazione di Bazin - si è avuto un effetto di moltiplicazione, ma in questo caso la soglia tra queste due forme espressive è stata attraversata con grande frequenza sia in un senso che nell'altro.

Il fenomeno di ricaduta del cinema sulla letteratura viene definito da Antonio Costa con l'efficace formula di 'effetto rebound' (Costa 45) e tra le sue prime vittime può annoverare nientemeno che lo stesso Pirandello (l'esempio più evidente è il suo Quaderni di Serafino Gubbio operatore). Ciò che è però interessante sottolineare è l'ancor più massiccio e sistematico saccheggio effettuato dal cinema nei confronti di opere letterarie. ${ }^{6}$ In particolare poi, quella che Costa definisce la "via del postmodernismo", consiste "nel riciclare le immagini usate in un nuovo contesto che ne cambi il significato, nel fare un uso ironico dell'immaginario dei mass-media o nell'immettere il gusto del meraviglioso ereditato dalla tradizione letteraria in meccanismi narrativi che ne accentuino l'estraniazione" (Costa 61).

Tra i letterati interessati al cinema (ed influenzati dal cinema) che hanno fatto ampio uso del succitato tipo di strategia narrativa spicca quindi senza dubbio la figura di Pier Paolo Pasolini; un artista talmente interessato alla settima arte da rendere difficile ogni tentativo di tracciare una linea di demarcazione tra la sua attività filmica e quella letteraria. Già dalla metà degli anni Sessanta, infatti, l'opera pasoliniana era diventata infatti "qualcosa di assolutamente unitario, dove un libro di saggi poteva rimandare a una raccolta di poesie, un film poteva rinviare ad un intervento critico, e il vero testo che Pasolini offriva al lettore era in realtà l'insieme di tutti i suoi testi, integrati dal corpo stesso dell'autore" (Siti $45)^{7}$ dato che non c'era lavoro, tra gli ultimi, che non annettesse "lo spessore biografico dell'autore tra gli elementi formali della scrittura" (Siti 45). Pasolini, quindi, si distingue per aver portato a livelli estremi non solo il collegamento tra scrittura letteraria e cinematografica, ma anche e soprattutto l'intuizione pirandelliana di "cinema in quanto arte dello spettacolo, con una propria ed inedita drammaturgia" (Termine 220).

Per cogliere appieno la portata dell'influenza pirandelliana sulla settima arte, e soprattutto su Pasolini, occorre però prima ricordare quelle che Manuela Gieri definisce "Pirandello's utterances on the cinema, in his revolutionary overturn of traditional theatrical and narrative strategies, and, above all, in his essay on humour, L'umorismo (On Humor, 1908)" (Gieri 10). L'umorismo pirandelliano, infatti, si qualifica come "a linguistic

6 Come ricorda ancora Costa, "il cinema ha saccheggiato in modo sistematico la letteratura traendone ispirazione per le storie da raccontare e traducendo in immagini, spesso per un pubblico analfabeta, classici e libri di successo" (Costa 45).

A questo proposito può essere particolarmente interessante considerare sotto questa luce la presenza di Pasolini in veste di attore (nei panni di un allievo di Giotto) ne /l Decameron [1971]. 
response to the specific psychological and existential condition modern man experienced and, to a certain extent, still experiences, a condition defined by specific motifs such as fragmentation, division, and thus alienation or estrangement. The 'Pirandellian mode' produces metadiscoursive narratives that are self-reflexive, eccentric, and hypercritical, and pursue a true repositioning of the various parties involved in the discoursive practice" (Gieri 11).

La rivolta pirandelliana nei confronti del modo di narrare verista (ed in generale della tradizione che lo precedeva) si sposa quindi perfettamente con la reazione pasoliniana al populismo neorealista, e con la volontà di proporre un superamento del Neorealismo cinematografico stesso. Quello che possiamo riscontrare in Pasolini, infatti, è ben di più di un generico 'pirandellismo' ed è forse definito meglio dalla formula proposta da Antonio Costa, quando parla di "disegno lucidamente tendenzioso, eretico" (Costa 128) - riecheggiando peraltro il titolo di uno degli scritti pasoliniani.

Nei vari saggi scritti sul cinema, Pasolini distingue tra un uso proprio ed uno pretestuale del discorso libero indiretto. II primo lo si ottiene quando l'autore s'immerge nell'animo del personaggio (adottandone la psicologia ma anche, e soprattutto, la lingua), e questo non solo quando viene riportato il discorso in forma diretta, ma anche nei momenti descrittivi, in cui a parlare è l'autore e non il personaggio. L'uso pretestaule del discorso libero indiretto si ha invece quando l'autore usa la lingua del personaggio come semplice pretesto per esprimere la sua Weltanschauung, come accade nel romanzo borghese.

Di conseguenza, ciò che Pasolini propone non può che essere il brillante frutto ibrido e liminale dello 'strappo' che mette in comunicazione due forme espressive come cinema e letteratura: la soggettiva libera indiretta. Quest'ultima non è altro che una inquadratura spuria, o meglio a metà strada - e quindi non immediatamente riconoscibile - tra una soggettiva (che nel linguaggio filmico corrisponde a quello che in letteratura è il discorso diretto, tra virgolette) ed una oggettiva. In questo modo - ossia "senza permettere allo spettatore di stabilire con certezza se quell'immagine corrisponda o meno a ciò che sta vedendo uno dei personaggi del film" (Manzoli 52) ed avvalendosi spesso anche di un certo tipo di messa a fuoco o di illuminazione - Pasolini non intende certo restituirci l'irraggiungibile soggettività dei personaggi, quanto piuttosto darci l'impressione di essere ancora una volta davanti ad uno 'strappo', ad un elemento che anzichè rafforzare l'autorità della narrazione la problematizza, lasciandoci intravedere quell'altrove, quella zona liminale tanto cara ai personaggi pirandelliani.

Nel cortometraggio Che cosa sono le nuvole [1968] poi Pasolini, oltre a mescolare profondità tragica e malinconica dell'assunto narrativo, ripropone il succitato stratagemma della soggettiva libera indiretta e, 
quasi verbatim, anche il tema dello 'strappo nel cielo di carta'. La trama ruota intorno a due marionette gettate in una discarica che, interrogandosi su cosa siano quelle nuvole che vedono per la prima volta, si presentano, secondo Piero Spila, come "umane e vulnerabili pedine, confuse davanti al grande mistero della vita" (Spila 67). Una scena, insomma, in cui ancora una volta emerge un'influenza profonda del pensiero pirandelliano, e che nel contempo si configura come un altro contributo all'esplorazione dell'identità umana fornito da Pasolini.

In conclusione, se lo 'strappo nel cielo di carta" era l'immagine con cui Pirandello aveva sottolineato la differenza fra la tragedia classica ed $i$ drammi dell'uomo moderno - anticipando, fra le altre cose, anche quello che dal 1916 in poi sarebbe stato il senso del suo teatro, rivoluzionario proprio perché giocato sull'interiorità dei protagonisti ${ }^{8}$ - ciò che all'uomo postmoderno rimane, non è altro che un cielo strappato al quale è possibile guardare, ma solo a patto di essere consci della propria problematica solitudine. Lo sguardo di Pasolini, quindi, da un lato si qualifica ancora una volta per la volontà di soffermarsi su una liminalità quanto mai fruttuosa, ma dall'altro pare spingerci a guardare con maggiore attenzione e ancora più a fondo in quel baratro che si apre dinanzi a nostri occhi; quasi a volerci spingere ad oltrepassare, finalmente, quella soglia ed a strappare il cielo di carta che grava sulle nostre coscienze di occidentali pasciuti e ottusi, facendoci vedere di che lacrime e di che quanto sangue gronda questa nostra "community without propinquity," secondo le parole del sociologo Melvin Webber.

\section{RIFERIMENTI BIBLIOGRAFICI}

Alonge, Roberto. Pirandello tra realismo e mistificazione. Napoli: Guida, 1972. Barilli, Renato. La linea Svevo-Pirandello. Milano: Mursia, 1972.

Barthes, Roland. The Pleasure of Text. Trans. Richard Miller. New York: Hill and Wang, 1975.

Biasin, Gian Paolo. "Lo specchio di Moscarda", in Paragone, giugno 1972.

Costa, Antonio. Immagine di un'immagine. Cinema e letteratura. Torino: UTET, 1993.

Debenedetti, Giacomo. II romanzo del novecento. Milano: Garzanti, 1971.

Dombroski, Robert. Le totalità dell'artificio. Ideologia e forma nel romanzo di Pirandello. Padova: Liviana Editrice, 1978.

Gieri, Manuela. Contemporary Italian Filmmaking. Strategies of Subversion.

8 Esattamente il contrario di quanto era accaduto fino a quel momento, con i vari deus ex machina o anche solo con le 'grand narratives'. 
Pirandello, Fellini, Scola, and the Directors of the New Generation. Toronto: University of Toronto Press, 1995.

Lacan, Jacques. Écrits : A Selection. Trans. Alan Sheridan. New York: W. W. Norton, 1977.

Mansfield, Nick. Subjectivity. Theories of the Self from Freud to Haraway. New York: New York University Press, 2000.

Manzoli, Giacomo. Cinema e letteratura. Roma: Carocci Editore, 2003.

Ossani, Anna. "Pirandello nel linguaggio della scena" in Pirandello nel linguaggio della scena. Materiali bibliografici dai quotidiani italiani. (1962-1990). rado Donati e Anna Ossani (a cura di). Ravenna: Longo Editore, 1993.

Pirandello, Luigi. II fu Mattia Pascal. Milano: Mondolibri, 2003a.

---. Uno, nessuno e centomila. Milano: Mondolibri, 2003b.

Siti, Walter. "La sceneggiatura e la poetica del non-finito" Bianco \& Nero n. 1 (genn.- febbr. 1999). 41:45.

Spila, Piero. Pier Paolo Pasolini. Roma: Gremese Editore, 1999.

Stocchi Perucchio, Donatella. Pirandello and the Vagaries of Knowledge. A Reading of II fu Mattia Pascal. Saratoga: ANMA Libri and Dept. of French and Italian Stanford University, 1991.

Termine, Liborio. "Il cinema e la vergogna" in Fabri, Simonigh, e Termine. II cinema e la vergogna negli scritti di Verga, Bontempelli, Pirandello. Torino: Testo \& Immagine, 1998.

Weiss, Auréliu. Le Théâtre de Luigi Pirandello dans le mouvement dramatique contemporain. Paris : Librairie 73, 1965. 